\title{
Study of optical phonon modes of CdS nanoparticles using Raman spectroscopy
}

\author{
RAJEEV R PRABHU and M ABDUL KHADAR* \\ Department of Physics, Centre for Nanoscience and Nanotechnology, University of Kerala, \\ Kariavattom PO, Thiruvananthapuram 695 581, India
}

\begin{abstract}
The reduction in the grain size to nanometer range can bring about radical changes in almost all of the properties of semiconductors. CdS nanoparticles have attracted considerable scientific interest because they exhibit strongly size-dependent optical and electrical properties. In the case of nanostructured materials, confinement of optical phonons can produce noticeable changes in their vibrational spectra compared to those of bulk crystals. In this paper we report the study of optical phonon modes of nanoparticles of CdS using Raman spectroscopy. Nanoparticle sample for the present study was synthesized through chemical precipitation technique. The CdS nanoparticles were then subjected to heat treatment at low temperature $\left(150^{\circ} \mathrm{C}\right)$ for extended time intervals. The crystal structure and grain size of the samples were determined using X-ray diffraction and HRTEM. The Raman spectra of the as-prepared and heat treated samples were recorded using conventional Raman and micro-Raman techniques. The spectrum of as prepared sample exhibited an intense, broad peak at $301 \mathrm{~cm}^{-1}$ corresponding to the LO phonon mode. Higher order phonon modes were also observed in the spectra. A noticeable asymmetry in the Raman line shape indicated the effect of phonon confinement. Other features in the spectra are discussed in detail.
\end{abstract}

Keywords. Nanoparticles; optical phonons; cadmium sulfide; Raman.

\section{Introduction}

Semiconductor nanoparticles form an important class of materials because of their interesting physical properties and their technological applications (Brus 1986; Henglein 1989; Weller 1993; Alivisatos 1996). Quantum size effects exhibited by semiconducting nanoparticles have been at the centre of scientific interest for the past two decades. In these investigations, vibrational spectroscopy has played a vital role as quantum size effect which results from confinement of electrons, holes and phonons within the nanoparticles efficiently show up their signature in this type of spectroscopy. During the last few years, studies of quantum size effects are mainly focused on the observation of the shift and modification of the Raman peaks since, in addition to revealing the effects of finite size of the particles on the vibrational modes and exhibiting additional features arising from their large values for surface to volume ratio, it can probe the local vibrational environment.

Confinement of electrons, holes and vibrations to crystallites of size a few nanometers brings about dramatic changes in their optical properties (Murray et al 1993; Wang et al 2002). Confinement of charge carriers appears as a blue shift of the bandgap and can be observed in the absorption spectra. The coupling of confined electrons

*Author for correspondence (mabdulkhadar@rediffmail.com) and confined phonons is another factor that determines the optical properties of nanoparticles.

Only the zone centre optical phonons can be observed in ideal single crystals using Raman spectroscopy. But in nanocrystalline materials, $q \approx 0$ selection rule is relaxed due to interruption of lattice periodicity. It has been theoretically calculated and experimentally observed that as the particle size reduces, the Raman spectra develop marked asymmetry towards the low frequency side and exhibit a frequency shift also towards the low frequency side. In addition to the optical phonons, the acoustic phonons also are confined within the crystallites of nanostructured materials, when the grain sizes are very small (Nemanich et al 1981; Richter et al 1981; Duval et al 1986; Roy and Sood 1996). The confinement of acoustic phonons results in the appearance of frequencies which depend on the elastic properties of the material through longitudinal and transverse sound velocities. For particles of diameter $<10 \mathrm{~nm}$, the frequencies of these modes fall between 5 and $50 \mathrm{~cm}^{-1}$ and as a result, these modes are observable using Raman spectroscopy. The interesting feature of these frequencies is that they depend on the reciprocal of the particle size and this relation has been experimentally verified in several cases (Roy and Sood 1996).

Although the vibrational properties of nanoparticles have been extensively studied using conventional Raman spectroscopy, such samples have not been examined simultaneously using micro-Raman technique for probing the effect of high intensity of illumination in exciting Raman 
features of higher orders. In addition, even though the effect of high temperature heat treatment of nanoparticles of CdS on their Raman spectra have been extensively studied (Sivasubramanian et al 2006), such studies on CdS nanoparticles subjected to low temperature heat treatment for long periods have not been reported in the literature. In the present study, CdS nanoparticles are prepared using a simple chemical route. These particles are then heat treated in an attempt to study the effect of low temperature heat treatment on the crystal structure and other properties of the nanoparticle sample. The vibrational spectra of the as-prepared and heat treated samples are then recorded using conventional Raman spectroscopy as well as micro-Raman spectroscopy and these spectra are analysed.

\section{Experimental}

Nanoparticles of CdS were prepared in an aqueous medium through chemical precipitation technique starting from analar grade cadmium salt and sodium sulfide, and using triethanolamine as capping agent (Prabhu and Abdul Khadar 2005). The particles were then heat treated at $150^{\circ} \mathrm{C}$ in DMSO for 4,8 and $16 \mathrm{~h}$. The XRD patterns of the samples were recorded using Bruker diffractometer and $\mathrm{CuK} \alpha$ radiation. The as-prepared and heat treated samples were examined using JEOL-3010 HRTEM. The Raman spectra of these samples were recorded using SPEX Ramalog spectrometer using a $514 \mathrm{~nm}$ excitation line. Also the micro-Raman spectrum of a sample was re-

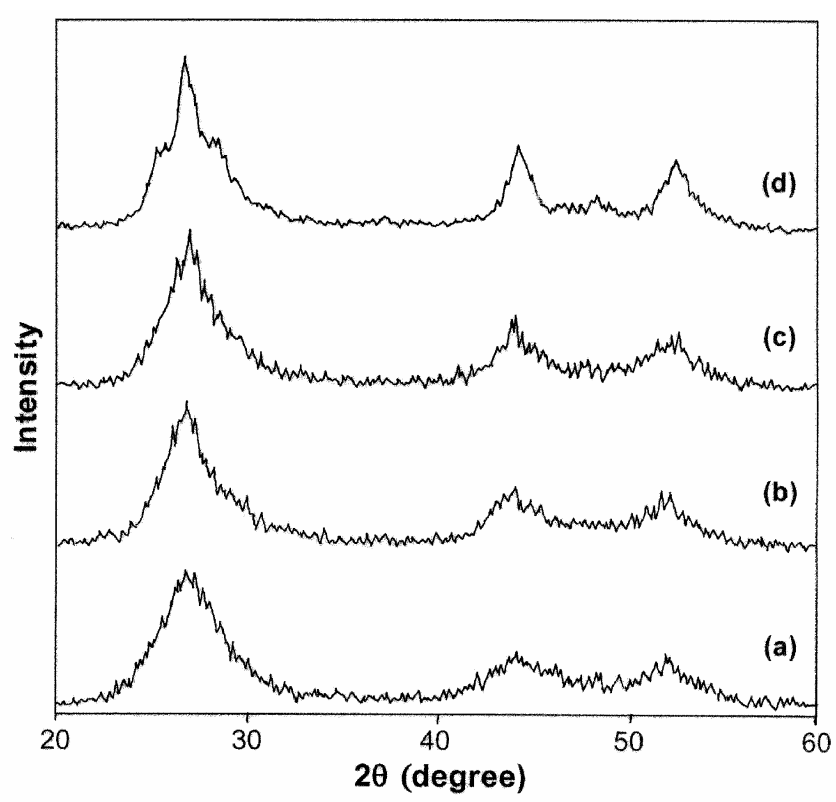

Figure 1. XRD patterns of nanoparticles of CdS: (a) as-prepared sample, (b) sample heat treated for $4 \mathrm{~h}$, (c) sample heat treated for $8 \mathrm{~h}$ and $(\mathrm{d})$ sample heat treated for $16 \mathrm{~h}$. corded using a Renishaw Ramascope Olympus DHZ-UMA spectrometer using a $514 \mathrm{~nm}$ excitation line.

\section{Results and discussion}

The XRD patterns of the as-prepared and heat treated samples of nanoparticles of $\mathrm{CdS}$ are shown in figure 1 . The XRD patterns of the CdS samples in figures 1a, b, c are identical and they exhibit broad peaks centred at $2 \theta$ values of $26.60,44.30$ and $52.03^{\circ}$ and a shoulder at $\sim 31.5^{\circ}$ which could be indexed to scattering from 111 , 220,311 and 200 planes, respectively of cubic CdS. The absence of peaks at 28.4 and $53^{\circ}$, which are associated only with hexagonal phase, eliminates the possibility of incorporation of hexagonal phase of $\mathrm{CdS}$ in the samples. When the duration of heat treatment is increased to $16 \mathrm{~h}$ partial conversion of $\mathrm{CdS}$ from cubic to hexagonal phase is identified (figure 1d). The grain size of the CdS nanoparticles is calculated for as-prepared and heat treated samples using the Scherrer equation (Cullity and Stock 2001) and is found to be $\sim 3 \mathrm{~nm}$. Low temperature annealing has not produced any grain growth. Hall-Williamson plot of as-prepared sample (figure 2) has a negative slope indicating compressive strain experienced by the particles.

Figure 3 shows HRTEM images of as prepared nanoparticles and those of the sample heat treated at $150^{\circ} \mathrm{C}$ for 8 and $16 \mathrm{~h}$. The images clearly show lattice fringes indicating good crystallinity of the samples. The TEM images give a grain size of $\sim 3 \mathrm{~nm}$ in agreement with XRD measurements.

The Raman spectra of the nanoparticle samples of CdS recorded in the 1 LO mode region are shown in figure 4. The $1 \mathrm{LO}$ peak centred around $300 \mathrm{~cm}^{-1}$ for the as prepared sample shows a small degree of asymmetry towards low frequency region. Although the positions of the

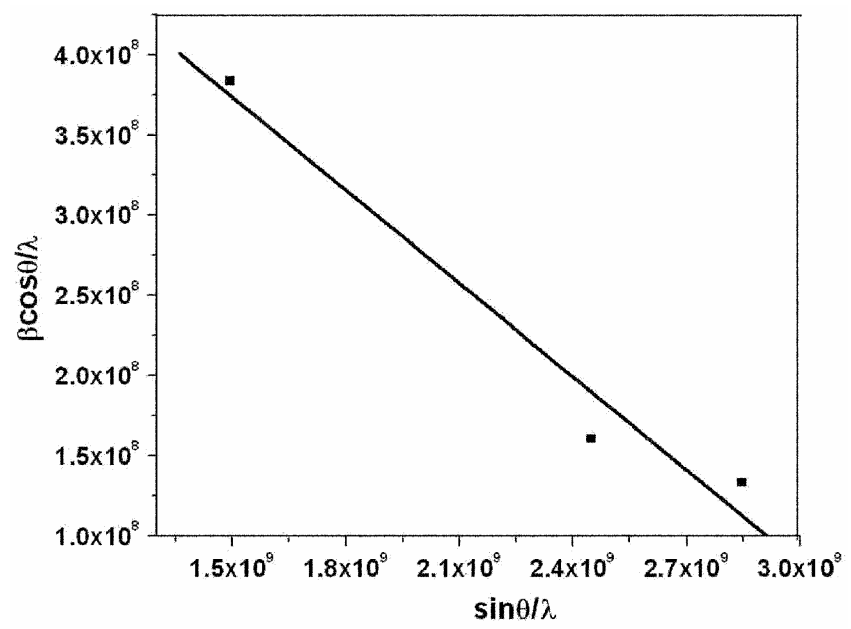

Figure 2. Hall-Williamson plot for as-prepared nanoparticles of CdS. 

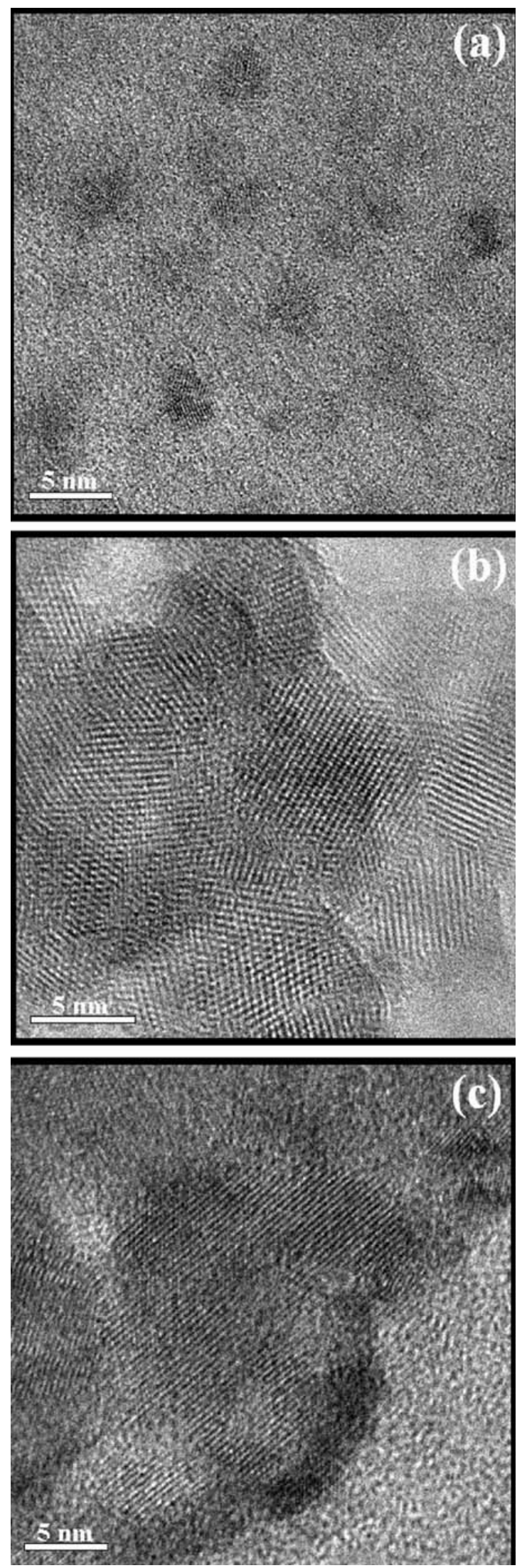

Figure 3. HRTEM images of nanoparticles of CdS: (a) asprepared CdS nanoparticles, (b) sample heat treated for $8 \mathrm{~h}$ and (c) sample heat treated for $16 \mathrm{~h}$. peaks are unchanged, the width (FWHM) decreased by noticeable amount on heating the samples. 1 LO peak of $\mathrm{CdS}$ is reported to appear around $300 \mathrm{~cm}^{-1}$ in micron and nanometer sized particles and is the prominent peak in all the reported results on CdS samples (Chuu et al 1991; Chuu and Dai 1992; Abdul Khadar and Thomas 1995; Nandakumar et al 2001). Figure 5 shows the microRaman spectrum of the CdS nanoparticles heat treated for $16 \mathrm{~h}$. The peaks are obtained at 301,603 and $909 \mathrm{~cm}^{-1}$ corresponding to $1 \mathrm{LO}, 2 \mathrm{LO}$ and 3 LO optical phonons, respectively.

From figure 4 it is seen that the first order LO Raman line is not only broadened, but also it shows an asymmetric broadening towards the low frequency side. The $1 \mathrm{LO}$ phonon frequency for a single crystal of $\mathrm{CdS}$ was reported as $305 \mathrm{~cm}^{-1}$ (Tell et al 1966), while the value obtained in the present study is around $300 \mathrm{~cm}^{-1}$. This frequency shift of the 1 LO Raman peak in CdS nanoparticles has been studied before and is mainly ascribed to the grain size effect (Chuu and Dai 1992). It is found from figure 4 that

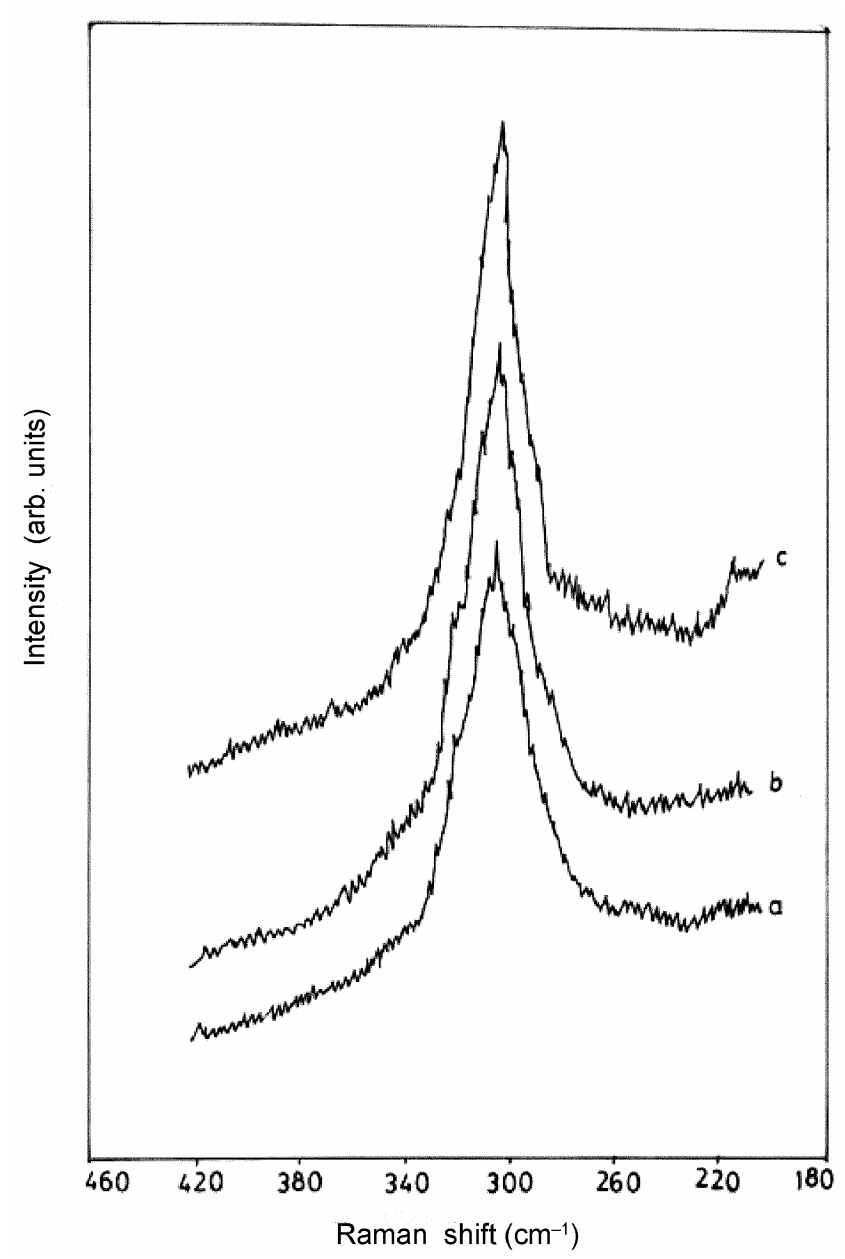

Figure 4. Raman spectra of CdS nanoparticles. a. Asprepared sample, b. sample heat treated for $4 \mathrm{~h}$ and c. sample heat treated for $8 \mathrm{~h}$. 
the position of these 1 LO peak does not undergo any shift for the heat treated samples but their FWHM has decreased noticeably compared to that of the as-prepared sample. It is now well known that confinement of phonons, optical as well as acoustic, influences the phonon spectra conspicuously when the grain size falls to a few nanometers. Confinement of optical phonons causes an asymmetry in the line shape and a shift towards the low frequency side compared to that for bulk CdS. In bulk crystals, the phonon eigen state is a plane wave and the selection rule for Raman scattering is $q \approx 0$, where $q$ is the wave vector. But in nanocrystallites, the spatial correlation function of the phonons is finite due to the phonon confinement, and hence $q \approx 0$ selection rule is relaxed and Raman signals from the phonon branch away from the zone centre also contribute to the resultant Raman line. The net effect of this contribution is the development of a marked asymmetry of the Raman line towards the low frequency side and a shift in the peak position which also is towards the low frequency side. Quantitative descriptions of the effect of phonon confinement on the Raman spectra of nanostructured materials including $\mathrm{CdS}$ (Arora et al 2004) have been reported by different researchers (Richter et al 1981; Campbell and Fauchet 1985).

In nanoparticles, the surface layers occupy a large percentage of the total volume of the material. It is known that the atomic arrangement on and near the grain boundaries is far from equilibrium and hence the Raman signals from these layers may be more similar to those from amorphous structures than those from crystalline ones (Bobovich 1988). If these surface layers of the nanoparticles have a chance to acquire a more ordered arrangement, then their contribution to the width of the Raman lines should decrease. Figure 4 shows that, the FWHM of the Raman lines noticeably decreases on increasing the duration of heating. It may be noted that, in

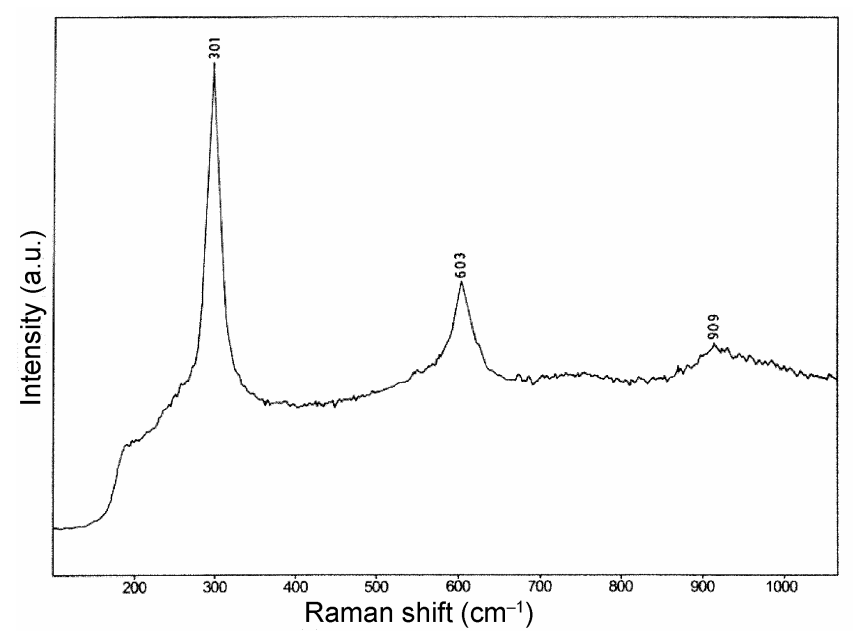

Figure 5. Micro-Raman spectrum of CdS nanoparticles heat treated for $16 \mathrm{~h}$. this study, the temperature of heat treatment is kept low $\left(150^{\circ} \mathrm{C}\right)$ to prevent grain growth. As seen from the XRD patterns in figure 1, the grain sizes of the particles have not changed appreciably due to heat treatment but the heat treatment has caused an improvement of the crystallinity of the nanoparticles and also a partial phase change (figure 1d). This leads to the inference that the heat treatment of the samples have caused a more regular arrangement of the atoms in the surface layers of the nanoparticles and to a partial change in the crystal structure and reduction of defect density. The X-ray diffraction patterns (figure 1d) and HRTEM pictures (figure 3c) clearly indicate a partial change of phase on heat treatment of the sample. The decrease in the FWHM of the 1 LO Raman line with heat treatment may be due to this partial change in the crystal structure and reduction of defect density. The broadening of the spectra, the asymmetry of the line shape and the shift of the peak towards lower frequency may be attributed to optical phonon confinement (Arora et al 2007).

Leite et al (1969) reported intensity measurements of $n$th order Raman scattering features $(n=1,2 \ldots 9)$ in $\mathrm{CdS}$ crystals as a function of temperature and laser frequency. The lines higher than second-order disappeared when the laser frequency was far below $E_{\mathrm{g}} / \hbar$, where $E_{\mathrm{g}}$ is the bandgap. Abdi et al (2006) recently reported three orders of Raman peaks associated with LO phonons of $\mathrm{CdS}$ nanorods at 301, 603 and $906 \mathrm{~cm}^{-1}$ when excited with laser light of wavelength, $496 \mathrm{~nm}$. Bischof et al (1997) have studied the intensity dependent micro-Raman spectra of $\mathrm{CdS}_{x} \mathrm{Se}_{1-x}$ nanocrystals embedded in borosilicate glass matrix and found a linear dependence of the Raman signal on laser intensity under weak laser excitation while the Raman intensity deviated from the linear behaviour for high laser intensities. In the present study, we observed that the micro-Raman spectra of nanoparticles of CdS clearly showed first, second and third order LO Raman peaks when excited using laser light of wavelength, $514 \mathrm{~nm}$. XRD and HRTEM results indicate a grain size of $\sim 5 \mathrm{~nm}$ for the particles of CdS which corresponds to a bulk bandgap of $\sim 2.4 \mathrm{eV}$. The energy of the laser (wavelength $514 \mathrm{~nm}$ ) is close to the bandgap of CdS nanoparticles and the excitation of three orders of the longitudinal optical phonon modes in the Raman scattering spectrum of nanoparticles of $\mathrm{CdS}$ in the present study is attributed to resonance effects or high laser power intensity in the micro-Raman experiment or both.

\section{Conclusions}

Nanoparticles of CdS are synthesized through chemical precipitation technique. The particles are heat treated at $150^{\circ} \mathrm{C}$ for different time durations. The particles are characterized by XRD and HRTEM. Dispersive Raman and micro-Raman spectra of the samples are recorded and 
analysed. A marked asymmetry in the Raman line shape indicated the effect of phonon confinement. The microRaman spectra of nanoparticles of CdS clearly show first, second and third order LO Raman peaks when excited using laser light of wavelength, $514 \mathrm{~nm}$. The excitation of three orders of the longitudinal optical phonon modes in the Raman scattering spectrum of nanoparticles of $\mathrm{CdS}$ in the present study is attributed to resonance effects.

\section{Acknowledgements}

One of the authors (MAK) expresses his gratitude to the DST for sanctioning a project under NSTI-Phase II (No. SR/S5/NM-32/2003). (RRP) is thankful to the DST for granting a research fellowship. The authors are thankful to SAIF, IIT Madras, Chennai, for HRTEM measurements and to the Indian Diamond Institute, Surat, for microRaman measurement.

\section{References}

Abdi A, Titova L V, Smith L M, Jackson H E, Rice J M Y, Lensch J L and Lauhon L J 2006 Appl. Phys. Lett. 88043118 Abdul Khadar M and Thomas B 1995 Nanostruct. Mater. 5289 Alivisatos A P 1996 Science 271933

Arora A K, Rajalakshmi M and Ravindran T R 2004 in Encyclopedia of nanoscience and technology (ed.) H S Nalwa (Los Angeles: American Scientific Publishers) Vol. 8, p. 499

Arora A K, Rajalakshmi M, Ravindran T R and Subramanian V 2007 J. Raman Spectrosc. 38604
Bischof T, Lermann G, Schreder B, Materny A, Kiefer W and Ivanda M 1997 J. Opt. Soc. Am. B14 3334

Bobovich Ya S 1988 J. Appl. Spectrosc. (USA) 49869

Brus L 1986 J. Phys. Chem. 902555

Campbell H and Fauchet P M 1986 Solid State Commun. 58 739

Cullity B D and Stock S R 2001 Elements of X-ray diffraction (New York: Prentice Hall Publishers) 3rd ed., p. 664

Chuu D R and Dai C M 1992 Phys. Rev. B45 11805

Chuu D R, Dai C M, Hsieh W F and Tsai C T 1991 J. Appl. Phys. 698402

Duval S, Boukenter A and Champagnom B 1986 Phys. Rev. Letts 562052

Henglein A 1989 Chem. Rev. 891861

Leite R C C, Scott J F and Damen T C 1969 Phys. Rev. Letts 22 780

Murray C B, Norris D J and Bawendi M G 1993 J. Am. Chem. Soc. 1158706

Nemanich J, Solin S A and Martin R M 1981 Phys. Rev. B23 6348

Nandakumar P, Vijayan C, Rajalakshmi M, Arora A K and Murti Y V G S 2001 Physica E11 377

Prabhu Rajeev R and Abdul Khadar M 2005 Pramana-J. Phys. 65801

Richter H, Wang Z P and Ley L 1981 Solid State Commun. 39 625

Roy A and Sood A K 1996 Phys. Rev. B53 12127

Sivasubramanian V, Arora A K, Premila M, Sundar C S and Sastry V S 2006 Physica E31 93

Tell B, Damen T C and Porto S P S 1966 Phys. Rev. 144771

Wang W, Germanenko I and Shall M S E 2002 Chem. Mater. 143028

Weller H 1993 Adv. Mater. 588 